\title{
Guideline zur Axilladissektion
}

\author{
M. Zuber ${ }^{1}$, D. Oertli ${ }^{1}$, W. R. Marti ${ }^{1}$, T. Kocher ${ }^{1}$, A. Wildisen ${ }^{1}$, G. Berclaz ${ }^{2}$, O. R. Köchli ${ }^{2}$, F. Harder ${ }^{1}$ \\ Arbeitsgruppe «Guideline Mammakarzinom, Axilladissektion» *
}

\section{Kurzfassung}

1. Die einzige aussagekräftige Staging-Methode ist derzeit die chirurgische Lymphknotenentfernung mit histopathologischer Aufarbeitung. Der axilläre Nodalstatus beeinflusst die Prognose und den Entscheid zur adjuvanten Therapie.

2. 10 resezierte und histologisch untersuchte Lymphknoten aus den axillären Level I + II sind für eine zuverlässige Staging-Aussage notwendig; damit werden 97\% der Patientinnen in das korrekte Lymphknotenstadium eingeteilt.

3. Aus der axillären Lymphknotenresektion von Level I + II resultiert eine nicht zu vernachlässigende Morbidität.

4. Nach Validierung der «sentinel lymph node (SLN)»-Methode sollen Patientinnen zunächst in prospektive Studien eingeschlossen werden.

5. Die chirurgische Entfernung axillärer Lymphknoten reduziert das axilläre Rezidivrisiko.

6. Es gibt Hinweise, dass die axilläre Dissektion einen marginal positiven Einfluss auf das Überleben haben könnte.

7. Patientinnen müssen auf die Häufigkeit und den Schweregrad möglicher postoperativer Folgen der Axilladissektion aufmerksam gemacht werden.

8. Bei klinisch unauffälliger Axilla kann auf die Lymphadenektomie nur verzichtet werden, wenn die zusätzliche Staging-Information zum vornherein keine therapeutischen Konsequenzen hat.

9. Patientinnen, die im Rahmen von kontrollierten wissenschaftlichen Studien behandelt und nachgesorgt werden, weisen einen Überlebensvorteil auf.

\footnotetext{
* Weitere Experten:

J. Benz ${ }^{2}$, V. Dupont Lampert ${ }^{1}$,

E. Eicher ${ }^{3}$ (Leitung), U. T. Laffer ${ }^{1}$,

J. G. Poell ${ }^{4}$, M. Trutmann ${ }^{5}$

1 Schweizerische Gesellschaft für Chirurgie

2 Schweizerische Gesellschaft für Gynäkologie und Geburtshilfe 3 FMH (Leitung)

4 Schweizerische Gesellschaft für Plastische, Rekonstruktive und Ästhetische Chirurgie

5 Schweizerische Ärztezeitung
}

Korrespondenz:

PD Dr. med. M. Zuber Departement Chirurgie

Kantonsspital Olten

CH-4600 Olten

E-Mail: mzuber_ol@spital.ktso.ch

\section{Axilladissektion und Staging}

Bedeutung des axillären Lymphknotenstatus

1. Die einzige aussagekräftige StagingMethode ist derzeit die chirurgische Lymphknotenentfernung mit histopathologischer Aufarbeitung. Der axilläre Nodalstatus beeinflusst die Prognose und den Entscheid zur adjuvanten Therapie.

Der axilläre Lymphknotenstatus ist der wichtigste prognostische Faktor des invasiven Mammakarzinoms [1: $\left.\varepsilon_{3}\right]$.

Zwei Langzeitstudien zeigten eine klare Korrelation zwischen Überlebensrate und histo- logisch positiven oder negativen Lymphknoten $\left[2,3: \varepsilon_{3}\right]$.

Die klinische Beurteilung der Axilla ist nicht genügend zuverlässig. Dies zeigte die NSABP-B04-Studie mit einer hohen Rate falsch positiver (27\%) und falsch negativer (39\%) Lymphknoten [4: $\left.\varepsilon_{3}\right]$.

Bildgebende Verfahren sind noch nicht in der Lage, zuverlässig den axillären Nodalstatus zu etablieren. Weder die Nachweismethode mit Technetium-Sestamibi-Szintigraphie noch diejenige mit Positronen-Emissions-Tomographie (PET) zeigten bisher eine genügende diagnostische Treffsicherheit mit entsprechender Sensitivität, Spezifität und negativem prädiktivem Wert [5-10: $\left.\varepsilon_{3}\right]$ im Vergleich mit der Histologie. 


\section{Grade der Evidenz}

Grad I: Die Evidenz ist aufgrund randomisierter kontrollierter Studien (oder Meta-Analysen) von genügendem Umfang derart, dass die Gefahr, dass sie falsch positive oder falsch negative Resultate beinhalten, gering ist.

Grad II: Die Evidenz basiert auf randomisierten kontrollierten Studien, welche jedoch zu klein sind, um ihnen Grad I zuzusprechen; sie können positive Trends, welche jedoch nicht signifikant sind, oder gar keine Trends zeigen. Sie sind mit einem hohen Risiko falsch negativer Resultate verbunden.

Grad III: Die Evidenz basiert auf nicht randomisierten Kontroll- oder Kohortenstudien, Fallserien, Fallkontrollstudien oder Querschnittsstudien (die beiden letzteren Studientypen wurden nicht berücksichtigt).

Grad IV: Die Evidenz beruht auf der Meinung angesehener ExpertInnen oder Expertengremien, wie sie in publizierten Konsenskonferenzen oder in Guidelines angegeben werden.

Grad V: Die Evidenz basiert auf der Meinung derjenigen Personen, welche diese Guidelines geschrieben oder aktualisiert haben, beruhend auf ihrer Erfahrung, ihrer Kenntnis der einschlägigen Literatur und der Diskussion mit ihren Fachkollegen.

Die Evidenzgrade der einzelnen Aussagen sind mit $\varepsilon_{1}, \varepsilon_{2}, \varepsilon_{3}$ und $\varepsilon_{4}$ gekennzeichnet. Evidenzgrad V entspricht dem Konsens der Arbeitsgruppe und wird nicht speziell vermerkt.
Zur Zeit der Definition der Levels wurde angenommen, dass der Lymphabfluss sequentiell von Level I über II nach Level III verläuft. Direkte Metastasierungen in Level II und/oder III kommen vor. Dieses Überspringen von Niveaus wird Skip-Metastasierung genannt. Diese Metastasen sind selten (bis 4\%) [16: $\varepsilon_{3}$ ].

Um eine verlässliche Aussage betreffend Nodalstatus machen zu können, müssen mindestens 10 Lymphknoten reseziert und analysiert werden $\left[17,18: \varepsilon_{3}\right]$.

Mit der axillären Dissektion von Level I + II wird bei $97 \%$ der Patientinnen ein korrektes Staging erreicht $\left[19,20: \varepsilon_{3}\right]$.

\section{Chirurgisch-technisches Vorgehen der Axilladissektion Level I + II nach Berg}

Im Gegensatz zur Mastektomie wird bei der brusterhaltenden Operation eine separate Inzision (vertikal oder horizontal; cave Schnittführung wegen Kontrakturen!) in der Axilla gewählt. Der laterale Rand des M. pectoralis wird aufgesucht, und nach Schonung des Gefäss-Nerven-Bündels des N. pectoralis lateralis erfolgt die Identifikation der V. axillaris. Nicht tumorinfiltriertes axilläres Fettgewebe wird kranial der V. axillaris nicht reseziert. Das thorako-dorsale Gefässnervenbündel wird dargestellt und geschont. Im nächsten Schritt wird die laterale Thoraxwand freipräpariert, der N. thoracicus longus kann so in seiner Länge entlang der Thoraxwand identifiziert und geschont werden. Die Präparation des axillären Fettkörpers erfolgt von kranio-medial (ab medialer Begrenzung des M. pectoralis minor [Level II]) nach kaudal und lateral. Auch wird das Fettgewebe zwischen M. pectoralis major und minor mitreseziert. Unter Schonung des thorako-dorsalen GefässNerven-Bündels auf seiner ganzen Länge $(a b$ Höhe V. axillaris bis zu den Gefäss- und NervenAufzweigungen) kann das axilläre Fett bis auf den M. subscapularis und M. latissimus dorsi freipräpariert und reseziert werden. Je nach anatomischem Verlauf sollte mindestens ein interkosto-brachialer Nerv geschont werden. Drainagen werden in die freipräparierte Axilla plaziert.

Eine physiotherapeutische Anleitung zur Erlangung der seitengleichen Schultergelenksfunktion ist in den meisten Fällen nicht notwendig.

Anatomisch werden 3 Level des axillären Lymphknoten unterschieden:

1. Level I: latero-kaudal;

2. Level II: dorsal des M. pectoralis minor;

3. Level III: medio-kranial des M. pectoralis minor. 


\section{Bedeutung der axillären Lymphknoten Levels I - III nach Berg}

3. Aus der axillären Lymphknotenresektion von Level I + II resultiert eine nicht zu vernachlässigende Morbidität.

Wenn die Axilladissektion sich auf die Lymphknoten von Level I + II beschränkt, können Folgen der Axilladissektion wie Schmerzen, Sensibilitätsstörungen, Schultergelenkstörungen und Lymphödeme der oberen Extremität stark reduziert werden [21: $\varepsilon_{4}$ ].

Der alleinige Befall der Lymphknoten in Level III ist selten (4\%; vgl. oben Skip-Metastasen). Die Level-III-Ausräumung, die bei metastatischem Befall indiziert ist, ergab eine vermehrte Morbidität ohne zusätzliche Staging-Information [22: $\left.\varepsilon_{4}\right]$.

\section{«Sentinel Lymph Node» (SLN) -Verfahren als Staging-Methode}

Die Lektüre der internationalen Konsensus-Konferenz wird empfohlen [11, 12: $\left.\varepsilon_{4}\right]$.

4. Nach Validierung der SLN-Methode sollen Patientinnen zunächst in prospektive Studien eingeschlossen werden.

Das SLN-Verfahren erlaubt die Identifikation des primär drainierenden Lymphknotens der Axilla. Seine histologische Untersuchung, in der Regel im Schnellschnittverfahren, lässt Rückschlüsse auf die nachgeschalteten Lymphknoten der Axilla zu. Ist der SLN histologisch positiv, folgt die offene Axilladissektion von Level I + II nach Berg (vgl. oben). Damit stellt die SLN-Technik ein wichtiges Selektionsverfahren dar, das in der Mehrzahl der kleinen Primärtumoren eine offene Axilladissektion entbehrlich macht; dies sofern sich das Verfahren in Zukunft etabliert. Langzeitresultate sind ausstehend. Die längste publizierte mittlere Nachbeobachtungszeit nach SLN-Verfahren betrug 39 Monate. Nach dieser Zeit wurden bei 133 Patientinnen keine axilläre Rezidive beobachtet [23: $\varepsilon_{3}$ ].

Bevor das SLN-Verfahren in einer Institution eingeführt werden kann, bedarf es einer Validierungsphase; d.h., der SLN wird selektiv exzidiert und die Axilladissektion von Level I + II folgt unmittelbar. Die histologischen Befunde des SLN müssen mit jenen der non-SLN korreliert werden. Die SLN-Identifikationsrate sollte zwischen 95-100\%, die falsch negative Rate $\leq 5 \%$ sowie der negative prädiktive Wert nicht unter $95 \%$ liegen [24, 25: $\left.\varepsilon_{3}\right]$.
Vorerst sollten Patientinnen - nach Validierung der SLN-Methode in der jeweiligen Institution - in prospektive Studien eingeschlossen werden [24: $\varepsilon_{3} ; 11: \varepsilon_{4}$ ] (für die Schweiz: www. sentinelnode.ch) [26].

\section{Axilladissektion und axilläres (regionäres) Rezidiv}

5. Die chirurgische Entfernung axillärer Lymphknoten reduziert das axilläre Rezidivrisiko.

Lokoregionäre Tumormanifestationen kommen in der Axilla nach Brustchirurgie ohne Axillarevision relativ häufig vor. In einer retrospektiv durchgeführten Studie ist die 10-Jahres-Rezidivrate in der Axilla nach Tumorektomie ohne Axillarevision $28 \%$. Diese Rate ist abhängig von der Grösse des Primärtumors $(10 \%$ bei pT1 $\leq 1 \mathrm{~cm} ; 26 \%$ bei pT1c $>1-\leq 2 \mathrm{~cm} ; 33 \%$ bei pT $>2 \mathrm{~cm}$ ) [27: $\varepsilon_{3}$ ] (ohne axilläre Radiotherapie oder Systemtherapie). Eine prospektive Analyse ergab nach mittlerer Beobachtungszeit von 5 Jahren für prognostisch günstige Primärtumoren ( $<3 \mathrm{~cm}$; cN0, cM0, postmenopausal) eine Rezidivhäufigkeit von 6,7\% (pT1a und pT1b je um 2\%) [28: $\left.\varepsilon_{3}\right]$.

In der NSABP-B-04-Studie betrug die axilläre Rezidivrate bei Patientinnen mit klinisch nicht palpablen Lymphkoten, die ohne Axilladissektion mastektomiert worden waren $17,8 \%$. Die Mastektomie mit Axillarevision hingegen ergab bei Patientinnen mit negativen Lymphknoten eine axilläre Rezidivrate von $1,0 \%$, bei Patientinnen mit positiven Lymphknoten 3,1\% [29: $\left.\varepsilon_{1}\right]$.

Mehrere Studien bestätigten, dass die formale Axilladissektion das axilläre Rezidivrisiko stärker vermindert als das blinde Sampling weniger axillärer Lymphknoten [30: $\varepsilon_{3}$ ]. In einer Studie mit 3128 Patientinnen ohne klinisch palpable Lymphknoten betrug die 5-JahresWahrscheinlichkeit für ein axilläres Rezidiv 3\%, wenn $>5$ Lymphknoten entfernt wurden. Die 5-Jahres-Wahrscheinlichkeit beträgt dagegen 19\%, wenn keine Lymphknoten entfernt wurden. [31: $\varepsilon_{3}$ ]. Ähnliche Resultate publizierten auch andere Autoren [4: $\left.\varepsilon_{1} ; 32,33: \varepsilon_{3}\right]$. In der NSABP-B-04-Studie erlitt keine Patientin mit $\geq 6$ entfernten Lymphknoten ein axilläres Rezi$\operatorname{div}$ [29: $\varepsilon_{1}$ ]. Kjaergaard forderte aufgrund einer Untersuchung bei über 3000 Patientinnen 10 und mehr resezierte histologisch analysierte Lymphknoten, da nach mittlerer Beobachtungszeit von 24 Monaten die Rezidivrate $0 \%$ betrug [34: $\left.\varepsilon_{3}\right]$. 


\section{Axilladissektion und Überleben}

6. Es gibt Hinweise, dass die axilläre Dissektion einen marginal positiven Einfluss auf das Überleben haben könnte.

Da die Axilladissektion das axilläre Rezidivrisiko vermindert, könnte daraus auch geschlossen werden, dass sie das Überleben ebenfalls positiv beeinflusst [35: $\left.\varepsilon_{3}\right]$. Auf Grund bestehender Daten kann eine Verlängerung des Überlebens nicht angenommen werden.

In der NSABP-B-04-Studie wurden < 70jährige Patientinnen mit Frühkarzinomen und klinisch negativer Axilla in 3 verschiedene Therapiegruppen randomisiert: Die Patientinnen von 2 Gruppen mastektomiert mit, eine Gruppe mastektomiert ohne Axilladissektion [29: $\varepsilon_{2}$ ]. Das 10-Jahres-Überleben betrug 58\% für diejenigen mit Mastektomie und Axilladissektion und $54 \%$ für diejenigen mit alleiniger Mastektomie ( $p>0,05$ ). Eine randomisierte Studie mit $658 \mathrm{~Pa}-$ tientinnen mit Frühkarzinom zeigt einen positiven Effekt der Axilladissektion auf das Überleben [36: $\left.\varepsilon_{1}\right]$. Die Patientinnen wurden in eine Gruppe Tumorektomie mit Axilladissektion und Bestrahlung der Restbrustdrüse und in eine Gruppe mit Tumorektomie mit Nachbestrahlung der Restbrustdrüse und der Axilla randomisiert. Im 5-Jahres-Überleben zeigte sich ein kleiner, aber statistisch signifikanter Vorteil $(p=0,014)$ für die Patientinnen mit chirurgischer Axilladissektion (96,6\% versus 92,6\%). Es ist aber möglich, dass dieser kleine Vorteil dadurch verursacht wurde, dass in dieser Gruppe 11 Patientinnen zusätzlich mittels adjuvanter Chemotherapie behandelt wurden.

Aus der Serie von 3128 Patientinnen von Graversen et al. lässt sich ein Überlebensvorteil ableiten. Die Patientinnen, bei denen < 5 Lymphknoten aus der Axilla entfernt wurde, hatten eine erhöhte Axillarezidivrate und ein schlechteres 5-Jahres-Überleben als die Patientinnen mit ausgedehnterer Axillachirurgie [31: $\left.\varepsilon_{3}\right]$. Eine umfassende Review der bis 1995 publizierten Daten hatte den Schluss erlaubt, dass Hinweise aber keine Beweise dafür vorliegen, dass die initiale Axilladissektion oder sogar die axilläre Bestrahlung ein Langzeiteffekt bewirken kann, wenn auch in geringem Ausmass [37: $\left.\varepsilon_{3}\right]$.

Die Meta-Analyse von 6 randomisierten, kontrollierten Studien mit insgesamt 3000 Patientinnen bei denen die Brustchirurgie mit versus ohne Axilladissektion verglichen wurde, zeigte einen Überlebensvorteil für die Gruppe mit Axilladissektion von 5,4\% (Konfidenz-Intervall von $95 \%=2,7-8,0 \%$; Wahrscheinlichkeit des Überlebensvorteils von 99,5\%). Dabei gilt es aber zu beachten, dass in dieser Analyse zu wenig Patientinnen mit pT1a-Tumoren eingeschlossen worden waren, um diesen Schluss auf diese Untergruppe anwenden zu können [38: $\varepsilon_{4}$ ] Bei pT1a-Karzinomen war nach einer mittleren Beobachtungszeit von 7 Jahren das krankheitsfreie Überleben $85 \%$ und das Mammakarzinom-spezifische Überleben $95 \%$ [39: $\left.\varepsilon_{3}\right]$.

\section{Axilladissektion und Morbidität}

7. Patientinnen müssen auf die Häufigkeit und den Schweregrad möglicher postoperativer Folgen der Axilladissektion aufmerksam gemacht werden.

Nach Axilladissektion können Früh- und Spätkomplikationen auftreten. Häufigkeit und Schweregrad dieser Komplikationen hängen vom Ausmass der Axilladissektion ab [37: $\left.\varepsilon_{3}\right]$. Armprobleme nach Brustchirurgie (Schmerzen, Sensibilitätsstörung, Schultersteifigkeit, Lymphödem) sind häufiger bei gleichzeitiger axillärer Lymphadenektomie als bei Ablatio simplex. In einer 18-monatigen Follow-up-Studie war die durchschnittliche Häufigkeit von Armproblemen in der Gruppe mit Axilladissektion 2,5 pro Patientin im Vergleich zu 0,9 pro Patientin ohne Axilladissektion ( $p=0,0001)$ [40: $\varepsilon_{3}$ ].

Taubheitsgefühl und Dysästhesien an der dorsomedialen Seite des Oberarms können nach Verletzung interkostobrachialer Nerven auftreten, was fast als Regelfall anzusehen ist. In einer Untersuchung ein Jahr postoperativ hatten fast 80\% der Patientinnen ein Taubheitsgefühl im Versorgungsgebiet der interkostobrachialen Nerven $\left[41,42: \varepsilon_{3}\right.$ ]. In einer Untersuchung von 432 Patientinnen im Stadium I oder II, die 2-5 Jahre postoperativ tumorfrei waren, hatten $30 \%$ wiederholt Schmerzen in Brust, Axilla oder Thorax (17\% leichte, 11\% moderate und 2\% starke Schmerzen) [43: $\varepsilon_{3}$.

Mit einer gewissen Einschränkung der Schulterbeweglichkeit muss in 17\% der Fälle gerechnet werden [41: $\left.\varepsilon_{3}\right]$. Ein eingesteiftes Schultergelenk nach Axilladissektion ist heute extrem selten [44].

Das Lymphödem ist die folgenschwerste Komplikation nach Axilladissektion. Die Häufigkeit des Auftretens hängt vom Ausmass der Axilladissektion ab [45-47: $\varepsilon_{3}$ ]. Die publizierte Häufigkeit hängt auch von der verwendeten Definition des Lymphödems ab. Basiert die Definition auf subjektiven und objektiven Befunden ist die Häufigkeit des Lymphödems 11-27\% [41, 46, 
48, 49: $\varepsilon_{3}$ ] Wird hingegen das Lymphödem als «klinisch offensichtlich» definiert, sinkt die Häufigkeit auf $2-7 \%\left[46,50,51: \varepsilon_{3}\right]$. In einer Serie wird berichtet, dass bei $10 \%$ der Patientinnen ein objektiv beobachtbares, aber nicht schweres Lymphödem auftrat [52: $\left.\varepsilon_{3}\right]$.

Insgesamt sind postoperative Probleme nach Axilladissektion häufig. Über $80 \%$ der Patientinnen leiden an einer oder mehreren postoperativen Folgen. Zwei bis fünf Jahre postoperativ beklagen immer noch mehr als 50\% der Patientinnen Schmerzen, Taubheitsgefühl oder Kribbel-Parästhesien. Diese Symptome sind jedoch meist wenig ausgeprägt [43: $\varepsilon_{3}$ ].

Wundinfekte sind häufiger bei älteren, adipösen oder mangelernährten Frauen, wie auch bei längerer Verweildauer von Wunddrainagen, vorangegangener Brustchirurgie, nach Radiotherapie der Region, Biopsie oder wiederholten Serompunktionen $\left[53,54: \varepsilon_{3}\right]$. Eine perioperative Antibiotikaprophylaxe senkt die Wundinfektrate $\left[55: \varepsilon_{1}\right]$.

\section{Kann auf die Axilladissektion prinzipiell verzichtet werden?}

8. Bei klinisch unauffälliger Axilla kann auf die Lymphadenektomie nur verzichtet werden, wenn die zusätzliche Staging-Information von vornherein keine therapeutischen Konsequenzen hat.

\section{Staging kleiner Tumoren}

Die Inzidenz befallener axillärer Lymphknoten bei kleinen Tumoren $(\leq 1 \mathrm{~cm})$ variiert in der Literatur zwischen $10 \%$ und $18 \%\left[27,56: \varepsilon_{3}\right]$. pT1a-Tumoren $(\leq 0,5 \mathrm{~cm})$ weisen selten axilläre Lymphknotenmetastasen (3\%) auf [39: $\varepsilon_{3}$ ].

Bei jungen Patientinnen ( $<40$ Jahre) mit lymphatischer Gefässinvasion und einer Tumorgrösse von $<1 \mathrm{~cm}$ wird eine axilläre Dissektion empfohlen, da die Analyse von 850 Patientinnen einen signifikant höheren axillären Lymphknotenbefall ergab als bei älteren Frauen [57: $\varepsilon_{3}$.

Das Axillastaging ist ein integraler Bestandteil der brusterhaltenden Therapie. Wenn sich aus dem chirurgischen Axillastaging bei klinisch unauffälliger Axilla keine Konsequenzen für die adjuvante Therapie ergeben (Konsensus St. Gallen 2001 [11: $\varepsilon_{4}$ ], kann auf eine Axilladissektion verzichtet werden [58: $\varepsilon_{4}$.

\section{Staging bei Patientinnen $>\mathbf{7 5}$ Jahre}

Berechtigt ist die Frage, inwieweit bei älteren Patientinnen (> 75 Jahre) ein axilläres Staging Sinn macht. Oft wird hier auf die adjuvante Chemo- therapie verzichtet, oder es steht bereits präoperativ fest, dass keine weitere Therapie durchgeführt werden kann [59: $\left.\varepsilon_{3}\right]$. Nach einer mittleren Beobachtungszeit von 47 Monaten ergab sich kein Anhaltspunkt für eine axilläre Tumormanifestation. Bei diesen Patientinnen mit klinisch unauffälliger Axilla kann auf eine Axilladissektion verzichtet werden.

\section{Klinische Studienprotokolle}

9. Patientinnen, die im Rahmen von kontrollierten wissenschaftlichen Studien behandelt und nachgesorgt werden, weisen einen Überlebensvorteil auf.

Den Patientinnen sollte die Möglichkeit zur Teilnahme an kontrollierten klinischen Studien immer offeriert werden. Bisherige Therapieentscheidungen basierten oft auf einer ungenügenden Datenevidenz ( $\varepsilon_{3}$ oder schwächer). Es gilt deshalb, dies zu verbessern und die Evidenz von Studien auf Level I oder II zu erhöhen. Dieses Ziel kann nur mit Hilfe gut konzipierter randomisierter und kontrollierter Studien erreicht werden. Hierhin gehört auch die Feststellung, dass Patientinnen, welche im Rahmen von Studien behandelt werden, ein besseres Überleben aufweisen als Patientinnen mit gleichen Tumorstadien ausserhalb von Studienprotokollen [60: $\left.\varepsilon_{3}\right]$. Ärzte, welche Patientinnen mit Mammakarzinom behandeln, sollten über laufende Studien und die Möglichkeit, daran teilzunehmen, orientiert sein.

\section{Literatur}

1 Clark GM. Prognostic and predictive factors. In: Harris JR et al. (editors). Diseases of the breast. Lippincott; Williams and Wilkins: 2000. p. 489.

2 Carter CL et al. Relation of tumor size, lymph node status, and survival in 24740 breast cancer cases. Cancer 1989;63:181.

3 Wilsin RE et al. The 1982 national survey of carcinoma of the breast in the United States by the American College of Surgeons. Surg Gynecol Obstet 1984;159:309.

4 Fisher B et al. The accuracy of clinical nodal staging and of limited axillary dissection as a determinant of histologic and nodal status in carcinoma of the breast. Surg Gynecol Obstet 1981;152:765.

5 Avril $\mathrm{N}$ et al. Assessment of axillary lymph node involvement in breast cancer patients with positron emission tomography using radiolabeled 2-(fluorine-18)-fluoro-2-deocy-D-glucose. J Natl Cancer Inst 1996;88:1204-9. 
6 Taillefer R et al. Metastatic axillary lymph node technetium-99m-MIBI imaging in primary breast cancer. J Nucl Med 1998;39:459-64.

7 Smith IC et al. Staging of the axilla in breast cancer. Ann Surg 1998;228:220-7.

8 Crippa F et al. Prospective evaluation of F-18-FDG PET in presurgical staging of the axilla in breast cancer. J Nucl Med 1998;39:4-8.

9 Guller U et al. Selective axillary surgery in breast cancer patients based on positron emission tomography with ${ }^{18} \mathrm{~F}$-fluoro-2-deoxy-D-glucose: not yet! Breast Cancer Res Treat 2002;71:171-3.

10 Yutani $\mathrm{K}$ et al. Comparison of FDG-PET with MIBI-SPECT in the detection of breast cancer and axillary lymph node metastasis. J Comput Assist Tomogr 2000;24:274-80.

11 Goldhirsch A et al. Meeting highlights: International consensus panel on the treatment of primary breast cancer. Seventh international conference on the adjuvant therapy of primary breast cancer. J Clin Oncol 2001;18:3817-27.

12 Schwartz GF et al. for the Consensus Conference Committee. Proceedings of the consensus conference on the role of sentinel lymph node biopsy in carcinoma of the breast, April 19-22, 2001, Philadelphia, Pennsylvania. Cancer 2002 94:2542-51.

13 Ravdin PM et al. Prediction of axillary lymph node status in breast cancer patients by use of prognostic indicators. J Natl Cancer Inst 1994; $86: 1771-5$

14 Menard S et al. Prognosis based on primary breas carcinoma instead of pathological nodal status. Br J Cancer 1994;70:709-12.

15 Gann PH et al. Factors associated with axillary lymph node metastasis from breast carcinoma: descriptive and predictive analyses. Cancer 1999; $86: 1511-9$

16 Veronesi U et al. Distribution of axillary node metastases by level of invasion. Cancer 1987; 59:682.

17 Kiricuta CI et al. A mathematical model of axillary lymph node involvement based on 1446 complete axillary dissections in patients with breast carcinoma. Cancer 1992;69:2496.

18 Axelsson CK et al. Axillary dissection for Level I and II lymph nodes is important in breast cancer classification. The Danish Breast Cancer Cooperative Group (DBCCG) Eur J Cancer 1992; 28:1415.

19 Danforth DN et al. Complete axillary lymph node dissection for stage I - II carcinoma of the breast. J Clin Oncol 1986;4:655.

20 Petrek JA et al. Axillary dissection: current practice and technique. Curr Probl Surg 1995;32:257.

21 Shibata HR. How important is a full axillary dissection: the case for surgery without full dissection. In: Wise L et al. (editors). Breast cancer: controversies in management. Armonk: Futura Publishing; 1994. p.177.

22 Ruffin WK et al. Rationale for routine axillary dissection in carcinoma of the breast. J Am Coll Surg 1995; 180:245.
23 Giuliano AE et al. Prospective observational study of sentinel lymphadenektomy without axillary dissection in patients with node-negative breast cancer. J Clin Oncol 2000;18:2553-9.

24 McMasters KM et al. Sentinel Lymph Node Biopsy for Breast Cancer - Not yet the Standard of Care. N Engl J Med 1998;339:990-5.

25 Miltenburg DM et al. Meta-analysis of sentinel lymph node biopsy in breast cancer. J Surg Res 1999;84:138-42.

26 Zuber M, Harder F. Mammakarzinom: Sentinel Lymph Node - Wächter intakter regionaler Lymphknoten. Praxis 2001;90:835-8.

27 Baxter $\mathrm{N}$ et al. Clinical behaviour of untreated axillary nodes after local treatment for primary breast cancer. Ann Surg Oncol 1996;3:235-40.

28 Greco $\mathrm{M}$ et al. Breast Cancer Patients treated without axillary surgery. Clinical Implications and biologic analysis. Ann Surg 2000;232:1-7.

29 Fisher B et al. Ten year results of a randomized clinical trial comparing radical mastectomy and total mastectomy with or without radiation. N Engl J Med 1985;312:674-81.

30 Macmillan RD et al. Sentinel node biopsy and axillary node sampling - how they compare. Eur J Surg Oncol 1998;24:618.

31 Graversen HP, et al. Breast cancer: Risk of axillary recurrence in node negative patients following partial dissection of the axilla. Eur J Surg Oncol 1988;14:407-12.

32 Sosa JA et al. Association between extent of axillary lymph node dissection and survival in patients with stage I breast cancer. Ann Surg Oncol 1998;5:140-9.

33 Fowble B et al. Frequencies, sites of relapse and outcome of regional node failures following conservative surgery and radiation for early breast cancer. Int J Radiat Oncol Biol Phys 1989; 17:703-10.

34 Kjaergaard J et al. and the Danish Cancer Cooperative Group. Probability of false negative nodal staging in conjunction with partial axillary dissection in breast cancer. Br J Surg 1985;72:365-7.

35 Hayward J et al. The significance in local control in the primary treatment of breast cancer. Arch Surg 1987;122:1244-7.

36 Cabanes PA et al. Value of axillary dissection in addition to lumpectomy and radiotherapy in early breast cancer. Lancet 1992;339:1245-8.

37 Recht A et al. Axillary lymph nodes and breast cancer. Cancer 1995;76:1491-512.

38 Orr RK. The impact of prophylactic axillary node dissection on breast cancer survival - a Bayesian meta-analysis. Ann Surg Oncol 1999; 6:109-16.

39 Silverstein MJ et al. Axillary lymph node dissection for T1a breast carcinoma. Is it indicated? Cancer 1994;73:664-7.

40 Maunsell et al. Arm problems and psychological distress after surgery for breast cancer. Can J Surg 1993;36:315.

41 Lin et al. Impact of axillary lymph node dissection on the therapy of breast cancer patients. J Clin Oncol 1993;11:1536. 
42 Pain SJ, Purushotham AD. Lymphoedema following surgery for breast cancer. Br J Surg 2000;87:1128-41.

43 Warmuth et al. Complications of axillary lymph node dissection for carcinoma of the breast. Cancer 1998;83:1362.

44 Petrek et al. Axillary dissection: Current practice and technique. Curr Probl Surg 1995;32:257.

45 Aitken et al. Complications associated with mastectomy. Surg Clin North Am 1983;63:1331.

46 Kissin et al. Risk of lymphedema following the treatment of breast cancer. Br J Surg 1986;73:580.

47 Yeoh et al. Primary breast cancer. Complications of axillary surgery. Acta Radiol Oncol 1986; 25:105.

48 Ball et al. Radical axillary dissection in the staging and treatment of breast cancer. Ann R Coll Surg Engl 1992;74:126.

49 Hayward et al. A new combined approach to the conservative treatment of early breast cancer. 1984;95:270.

50 Osborn et al. Breast conservation in the treatment of early breast cancer. A 20-year follow-up. Cancer $1984 ; 53: 349$.

51 Veronesi et al. Comparing radical mastectomy with quadrantectomy, axillary dissection, and radiotherapy in patients with small cancers of the breast. N Engl J Med 1981;305:6.

52 Ivens et al. Assessment of morbidity from complete axillary dissection. Br J Cancer 1992;66:136.
53 Beatty et al. A prospective analysis of nosocomial wound infection after mastectomy. Arch Surg 1983;118:1421.

54 Say et al. A biostatistical evaluation of complications from mastectomy. Surg Gynaecol Obstet $1974 ; 138: 370$

55 Platt et al. Perioperative antibiotic prophylaxis for herniorrhaphy and breast surgery. N Engl J Med 1990;322:153

56 Rivandeneira et al. Predictive factors associated with axillary lymph node metastases in $\mathrm{T} 1 \mathrm{a}$ and T1b breast carcinomas: Analysis in more than 900 patients. J Am Coll Surg 2000;191:1-8.

57 Gajdos C et al. Lymphatic invasion, tumor size and age are independent predictors of axillary lymph node metastases in women with T1 breast cancer. Ann Surg 1999;230:692-6.

58 Orr RK et al. A cost-effectiveness analysis of axillary node dissection in postmenopausal women with estrogen receptor-positive breast cancer and clinically negative axillary nodes. Surgery 1999;126:568-76.

59 Naslund E et al. Breast cancer in women over 75 years: is axillary dissection always necessary? Eur J Surg 1996;162:867-71.

60 Gnant $\mathrm{N}$ et al. (ABCSG). Impact of participation in randomized clinical trials on survival of women with early stage breast cancer An analysis of 7985 patients. Proc ASCO J Clin Oncol 2000;19:74. 